J. KAJIWARA

KŌDAI MATH. SEM. REP

26 (1975), 258-266

\title{
DOMAIN WITH MANY VANISHING COHOMOLOGY SETS
}

\author{
Dedicated to Professor Yûsaku Komatu on his sixtieth birthday
}

By JOJI KAJIWARA

\section{Introduction.}

The aim of this paper is to generalize a previous result [10] of the author. Oka [12] proved that a domain of holomorphy in $\boldsymbol{C}^{n}$ is a Cousin-I domain. Oka [13] also proved that a Cousin-II distribution in a domain of holomorphy in $\boldsymbol{C}^{n}$ has an analytic solution if and only if it has a topological solution. Grauert [5] proved that the canonical mapping of $H^{1}\left(X, \mathcal{A}_{L}\right)$ in $H^{1}\left(X, \mathcal{C}_{L}\right)$ is bijective for a Stein space $X$ and a complex Lie group $L$ where $\mathcal{C}_{L}$ and $\mathcal{C}_{L}$ are, respectively, the sheaves over $X$ of all germs of holomorphic and continuous mappings in $L$. Roughly talking, many cohomology sets have the possibility of vanishing in a Stein space.

Conversely, by Cartain [2] and Behnke-Stein [1], a Cousin-I domain in $\boldsymbol{C}^{2}$ is always a domain of holomorphy. By Cartan $[3] \boldsymbol{C}^{3}-\{(0,0,0)\}$ is a Cousin-I domain which is not a domain of holomorphy. By Thullen [15] $D=\left\{\left(z_{1}, z_{2}\right) \in C^{2}\right.$; $\left.\left|z_{1}\right|<1,\left|z_{2}\right|<1\right\}-\{(0,0)\}$ is a Cousin-II domain which is not a domain of holomorphy. By a previous remark [6] of the author, the Thullen's domain $D$ is an example of a Cousin-II domain which satisfies $H^{1}\left(D, \mathcal{O}^{*}\right) \neq 0$ for the sheaf $\mathcal{O}^{*}$ of multiplicative groups of all germs of never vanishing holomorphic functions. By the previous result [11] of the author and Kazama, however, a subdomain $X$ of a two-dimensional Stein manifold is a Stein manifold if $X$ satisfies $H^{1}\left(X, \mathcal{A}_{L}\right)=0$ for a complex Lie group $L$. In the case of higher dimension, the author [10] proved that a subdomain $X$ of a Stein manifold $S$ with real one-codimensional smooth boundary is a Stein manifold, if, for an abelian complex Lie group $L, X$ satisfies $H^{1}\left(X \cap P, \mathcal{A}_{L}\right)=0$ for all analytic polydisc $P$ in $S$.

The aim of this paper is to prove that a subdomain $X$ of a Stein manifold $S$ with real one-codimensional smooth boundary is a Stein manifold if, for a complex Lie group $L, X$ satisfies $H^{1}\left(X \cap P, A_{L}\right)=0$ for all analytic polydisc $P$ in $S$. The above boundary condition for $X$ can not be omitted as the above Cartan's example $\boldsymbol{C}^{3}-\{(0,0,0)\}$ shows. Roughly talking, a subdomain of a Stein manifold with many vanishing cohomology sets is also a Stein manifold. This is the principle which the author wants to maintain. In the proof, we use Lemmata and methods used in [11] and [9]. In this occasion the author ex-

Received Oct. 1, 1973. 
presses his hearty gratitude to Professor Y. Komatu who gives his continuous and kind encouragements since 1957.

\section{$\S 1$. Monotonically increasing sequence of domains.}

Let $M$ be a complex manifold. If $\varphi$ is a locally biholomorphic mapping of a complex manifold $D$ in $M,(D, \varphi)$ is called an open set over $M$. Let $\left(D_{1}, \varphi_{1}\right)$ and $\left(D_{2}, \varphi_{2}\right)$ be open sets over $M$. If there is a holomorphic mapping $\tau$ of $\left(D_{1}, \varphi_{1}\right)$ in $\left(D_{2}, \varphi_{2}\right)$ such that $\varphi_{1}=\varphi_{2} \circ \tau$, we write $\left(D_{1}, \varphi_{1}\right)<\left(D_{2}, \varphi_{2}\right)$. By this relation the set of all open sets over $M$ forms a partially ordered set $\mathfrak{D}$. A sequence $\left\{\left(D_{p}, \varphi_{p}\right)\right.$; $p=1,2,3, \cdots\}$ of open sets over $M$ is called a monotonically increasing sequence of open sets over $M$ if $\left(D_{p}, \varphi_{p}\right)<\left(D_{p+1}, \varphi_{p+1}\right)$ for any $p$. Let $\left\{\left(D_{p}, \varphi_{p}\right) ; p=1,2,3, \cdots\right\}$ be a monotonically increasing sequence of domains over $M$. Then it is a monotonically increasing sequence in the partially ordered set $\mathfrak{D}$. In the previous paper [8] the author proved the unique existence of its supremum in $\mathscr{D}$ and called it the limıt of the sequence $\left\{\left(D_{p}, \varphi_{p}\right) ; p=1,2,3, \cdots\right\}$. Let $D$ be a complex manifold and $L$ be a complex Lie group. The sheaf over $D$ of all germs of holomorphic mappings in $L$ is denoted by $\mathcal{A}_{L}$. Let $\mathfrak{U}=\left\{U_{\imath} ; \imath \in I\right\}$ be an open covering of $D$. We define an element $\left\{g_{\imath j}\right\}$ of $Z^{1}\left(\mathfrak{H}, \mathcal{A}_{L}\right)$ by putting $g_{\imath j}=1$ in

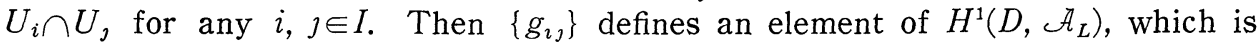
called a trivial element of $H^{1}\left(D, \mathcal{A}_{L}\right)$. If $H^{1}\left(D, \mathscr{A}_{L}\right)$ consists of only a trivial element, we write $H^{1}\left(D, A_{L}\right)=0$ for the sake of brevity.

A complex manifold $D$ is said to be analytically contractible if there is a continuous mapping $f(x, t)$ of $D \times[0,1]$ in $D$ such that $f(x, t)$ is a holomorphic mapping of $D$ in $D$ for any fixed $t \in[0,1]$, that $f(x, 0)$ is the identity mapping of $D$ and that $f(x, 1)$ is a constant mapping of $D$ in $D$.

LEMma 1. Let $\left\{\left(D_{p}, \varphi_{p}\right) ; p=1,2,3, \cdots\right\}$ be a monotonically increasing sequence of open sets over a Stern manifold $S$ such that each $D_{p}$ is analytrcally contractible and connected, $(D, \varphi)$ be its limit and $\tau_{p}$ be the canonical mapping of $D_{p}$ in $D$ for each $p$. Let $L$ be a complex Lie group and $\alpha$ be an element of $H^{1}\left(D, \mathcal{A}_{L}\right)$. If the image $\tau_{p}^{*}(\alpha)$ of $\alpha$ by the canonical mapping $\tau_{p}^{*}$ of $H^{1}\left(D, \mathcal{A}_{L}\right)$ in $H^{1}\left(D_{p}, A_{L}\right)$ induced by $\tau_{p}$ is a trivial element for any $p$, then $\alpha$ is a trivial element of $H^{1}\left(D, \mathcal{A}_{L}\right)$.

Proof. We denote by $\tau_{q}^{p}$ the canonical mapping of $D_{p}$ in $D_{q}$ for any $p$ and $q$ with $p \leqq q$. Let $\left\{Q_{p} ; p=1,2,3, \cdots\right\}$ be a sequence such that each $Q_{p}$ is a relatively compact subdomain of $D_{p}$, that $\tau_{p+1}^{p}\left(Q_{p}\right) \subset Q_{p+1}$ for any $p$ and that $D=\bigcup_{p=1}^{\infty} \tau_{p}\left(Q_{p}\right)$. Then $\left(Q_{p}, \varphi_{p} \mid Q_{p}\right)$ is a monotonically increasing sequence of domains over $S$ and $(D, \varphi)$ is its limit. Let $\left(\tilde{Q}_{p}, \tilde{\varphi}_{p}\right)$ be the envelope of holomorphy of $\left(Q_{p}, \varphi_{p} \mid Q_{p}\right)$ over $S$ and $\lambda_{p}$ be the canonical mapping of $Q_{p}$ in $\tilde{Q}_{p}$. For any $p$ and $q$ with $p \leqq q$, there is a holomorphic mapping $\tilde{\tau}_{q}^{p}$ of $\widetilde{Q}_{p}$ in $\widetilde{Q}_{q}$ such that $\tilde{\varphi}_{p}=\tilde{\varphi}_{q} \circ \tilde{\tau}_{q}^{p}$ and $\lambda_{q} \circ\left(\tau_{q}^{p} \mid Q_{p}\right)=\tilde{\tau}_{q}^{p} \circ \lambda_{p}$. Hence $\left\{\left(\tilde{Q}_{p}, \tilde{\varphi}_{p}\right)\right\}$ is a monotonically increasing sequence of domains over $S$. Let $(\tilde{D}, \tilde{\varphi})$ be its limit. Then $(\tilde{D}, \tilde{\varphi})$ is the 
envelope of holomorphy of $(D, \varphi)$. Let $\lambda$ be the canonical mapping of $D$ in $\tilde{D}$. For any $p$ there is a holomorphic mapping $\tilde{\tau}_{p}$ of $\tilde{Q}_{p}$ in $\tilde{D}$ such that $\tilde{\varphi}_{p}=\tilde{\varphi} \circ \tilde{\tau}_{p}$ and $\tilde{\varphi}_{p}=\tilde{\tau}_{q} \circ \tau_{q}^{p}$ for any $p$ and $q$ with $p \leqq q$. Since $\tilde{D}$ is a Stein manifold by Docquier-Grauert [4], there is a sequence $\left\{P_{p} ; p=1,2,3, \cdots\right\}$ of relatively compact analytic polycylinders $P_{p}$ defined by holomorphic functions in $\tilde{D}$ such that $P_{p}$ is a relatively compact subdomain of $P_{p+1}$ for any $p$ and that $\tilde{D}=\bigcup_{p=1}^{\infty} P_{p}$. Since $(\tilde{D}, \tilde{\varphi})$ is the limit of $\left(\tilde{Q}_{p}, \tilde{\varphi}_{p}\right)$, there is a sequence $\left\{\nu_{p} ; p=1,2,3, \cdots\right\}$ of positive integers such that $\tilde{\tau}_{\nu_{p}}$ maps a relatively compact subdomain $P_{p}^{\prime}$ of $Q_{\nu p}$ biholomorphically onto $P_{p}$ for any $p$. Without loss of generality, we may assume that $\nu_{p}=p$.

Now we go to prove Lemma 1. Let $\left\{f_{\imath \jmath}\right\}$ be an element of $Z^{1}\left(\mathfrak{H}, \mathcal{A}_{L}\right)$ for an open covering $\mathfrak{H}=\left\{U_{\imath} ; i \in I\right\}$ of $D$ such that $\left\{f_{\imath \jmath}\right\}$ is an element corresponding to $\alpha$. We put $\tau_{p}^{-1}(\mathfrak{H})=\left\{\tau_{p}^{-1}\left(U_{\imath}\right) ; \imath \in I\right\}$ for each $p$. Then $\tau_{p}^{-1}(\mathfrak{H})$ is an open covering of $D_{p}$ and $\left\{f_{\imath j} \circ \tau_{p}\right\}$ is an element of $Z^{1}\left(\tau_{p}^{-1}(\mathfrak{H}), \mathcal{A}_{L}\right)$. Since $\tau_{p}^{*}(\alpha)$ is trivial, there is an element $\left\{f_{\imath}^{p}\right\}$ of $C^{0}\left(\tau_{p}^{-1}(\mathfrak{H}), \mathcal{A}_{L}\right)$ for any $p$ such that

$$
f_{\imath j} \circ \tau_{p}=f_{j}^{p}\left(f_{\imath}^{p}\right)^{-1}
$$

in $\tau_{y}^{-1}\left(U_{i} \cap U_{\jmath}\right)$ for any $i, j \in I$. If we put

$$
f^{p}=\left(f_{\imath}^{p}\right)^{-1}\left(f_{\imath}^{p+1} \circ \tau_{p+1}^{p}\right)
$$

in $\tau_{p}^{-1}\left(U_{\imath}\right)$, then $f^{p}$ is a well-defined element of $H^{0}\left(D_{p}, \mathcal{A}_{L}\right)$. Since $D_{p}$ is analytically contractible, $H^{0}\left(D_{p}, \mathcal{A}_{L}\right)$ forms a connected topological group. Therefore, any neighborhood of the neutral element of $H^{0}\left(D_{p}, \mathcal{A}_{L}\right)$ generates $H^{0}\left(D_{p}, \mathcal{A}_{L}\right)$. Let exp be the exponential mapping of the Lie algebra $C^{m}$ of $L$ in $L$. There is a polydisc neighborhood $W$ of the origin of $C^{m}$ such that exp maps an open neighborhood of the closure of $W$ biholomorphically onto a neighborhood of 1 in $L$. We put $W^{\prime}=\exp (W)$. There is a finite set $\left\{f^{p, \nu}\right\}$ of holomorphic mappings $f^{p, \nu}$ of $D_{p}$ in $L$ for any $p$ such that

$$
f^{p}=\prod f^{p, \nu}
$$

and the $f^{p, \nu}\left(Q_{p}\right) \subset W^{\prime}$ for any $p$ and $\nu$. Then each $\left(\exp W^{\prime}\right)^{-1} \circ\left(f^{p, \nu} \mid Q_{p}\right)$ is a holomorphic mapping of $Q_{p}$ in $C^{m}$. Since $\left(\tilde{Q}_{p}, \tilde{\varphi}_{p}\right)$ is the envelope of holomorphy of $\left(Q_{p}, \varphi_{p} \mid Q_{p}\right)$, there is a holomorphic mapping $F^{p, \nu}$ of $\tilde{Q}_{p}$ in $C^{m}$ for any $p$ and $\nu$ such that

$$
\left(\exp W^{\prime}\right)^{-1} \circ\left(f^{p, \nu} \mid Q_{p}\right)=F^{p, \nu} \circ \lambda_{p} .
$$

Then $F^{p, \nu} \circ\left(\tilde{\tau}_{p} \mid P_{p}^{\prime}\right)^{-1}$ is holomorphic mapping of $P_{p}$ in $C^{m}$ for any $p$ and $\nu$. Let $\left\{\varepsilon_{p} ; p=1,2,3, \cdots\right\}$ be a sequence of positive numbers. Since $P_{p}$ is holomorphically convex with respect to $\tilde{D}$, there is a holomorphic mapping $G^{p, \nu}$ of $D$ in $C^{m}$ for any $p$ and $\nu$ such that

$$
\left|F^{p} \circ\left(\tilde{\tau}_{p} \mid P_{p}^{\prime}\right)^{-1}-G^{p, \nu}\right|<\varepsilon_{p}
$$


in $P_{p-1}$ for any $p$ and $\nu$. We put

$$
g^{p}=\Pi \exp \left(G^{p, \nu} \circ \lambda\right)
$$

in $D$ for any $p$. Then $g^{p}$ is a holomorphic mapping of $D$ in $L$ which approximates $f^{p}$ in some sense. We put

$$
g_{i}^{p}=f_{i}^{p}\left(g^{p-1} \circ \tau_{p}\right)\left(g^{p-2} \circ \tau_{p}\right) \cdots\left(g^{1} \circ \tau_{p}\right)
$$

in $\tau_{p}^{-1}\left(U_{\imath}\right)$. Then $\left\{g_{\imath}^{p} ; p=1,2,3, \cdots\right\}$ converges to a holomorphic mapping $g_{\imath}$ of $U_{\imath}$ in $L$ uniformly in any compact subset of $U_{\imath}$ if $\left\{\varepsilon_{p}\right\}$ is sufficiently small and decreasing. Then $\left\{g_{\imath}\right\} \in C^{0}\left(\mathfrak{H}, \mathcal{A}_{L}\right)$ satisfies

$$
f_{\imath j}=g_{j} g_{\imath}^{-1}
$$

in $U_{i} \cap U$, for any $\imath, \jmath \in I$.

Q.E. D.

\section{§2. Domains exhausted by L-regular domains.}

In the following Lemmata 2 and 3, we put

and

$$
U_{1}=\left\{\left(z_{1}, z_{2}\right) \in C^{2} ; 0<\left|z_{1}\right|<1,\left|z_{2}\right|<1\right\}
$$

$$
U_{2}=\left\{\left(z_{1}, z_{2}\right) \in C^{2} ;\left|z_{1}\right|<1,0<\left|z_{2}\right|<1\right\} \text {. }
$$

The following Lemmata 2 and 3 are, respectively, Lemmata 6 and 10 in the previous paper [11], so the proofs are omitted.

LeMma 2. Let $B$ be an $(m, m)$-matrix with a non-zero eigen-value. There are not $g_{i} \in H^{0}\left(U_{\imath}, \mathcal{A}_{G L(m, c)}\right)(\imath=1,2)$ such that

in $U_{1} \cap U_{2}$.

$$
\exp \left(\frac{B}{z_{1} z_{2}}\right)=g_{2} g_{1}^{-1}
$$

LEMMA 3. Let $B$ be a non-zero $(m, m)$-matrix, whose elgen-values are all zero. There are not $g_{i} \in H^{0}\left(U_{\imath}, \mathcal{A}_{G L(m, c)}\right)$ such that

$$
\exp \left(\exp \left(\frac{1}{z_{1}}+\frac{1}{z_{2}}\right) B\right)=g_{2} g_{1}^{-1}
$$

in $U_{1} \cap U_{2}$.

A complex manifold $P$ is called an analytic polydisc if there is a biholomorphic mapping of a polydisc $\left\{w=\left(w_{1}, w_{2}, \cdots, w_{n}\right) ;\left|w_{1}\right|<r_{1},\left|w_{2}\right|<r_{2}, \cdots,\left|w_{s}\right|<r_{s}\right\}$ $(0 \leqq s \leqq n)$ onto $P$. An analytic polydisc $P$ is analytically contractible and $H^{1}\left(P, \mathcal{A}_{L}\right)$ $=0$ for any complex Lie group $L$. Let $D$ be an open subset of a complex manifold $M$ and $L$ be a complex Lie group. If $H^{1}\left(D \cap P, A_{L}\right)=0$ for any analytic polydisc $P$ in $M, D$ is called an L-regular open set in $M$. A domain $D$ in a complex manifold $M$ is said to be exhausted by L-regular open sets if there is a 
sequence $\left\{D_{p}\right\}$ of $L$-regular open sets $D_{p}$ in $M$ such that $D_{p}$ is relatively compact open subset of $D_{p+1}$ for any $p$ and $D=\bigcup_{p=1}^{\infty} D_{p}$.

LEMMA 4. Let $L$ be a complex Lie group whose dimension $m$ is positive. Let $\Omega$ be a domain in $\boldsymbol{C}^{n}$ exhausted by L-regular domains $\Omega_{p}$ in $\boldsymbol{C}^{n}$. Then $\Omega$ is a Stein manifold.

Proof. In case that $n=1$, there is nothing to prove. In case that $n=2$, each $\Omega_{p}$ and, therefore, $\Omega$ is a Stein manifold by the previous paper [11]. So we may assume that $n \geqq 3$. It suffices to prove that $\Omega$ is $p_{7}$-convex in the sense of Docquier-Grauert [4] by Oka [14]. Assume that $\Omega$ were not $p_{7}$-convex. For any positive numbers $\varepsilon$ and $\varepsilon^{\prime}$ with $1>\varepsilon>\varepsilon^{\prime}$ we put

$$
\begin{aligned}
& D(\varepsilon)=\left\{w=\left(w_{1}, w_{2}, \cdots, w_{n}\right) \in \boldsymbol{C}^{n} ;\left|w_{1}\right|<1+\varepsilon,\left|w_{\imath}\right|<1(\imath=2,3, \cdots, n)\right\} \\
& \cup\left\{w=\left(w_{1}, w_{2}, \cdots, w_{n}\right) \in \boldsymbol{C}^{n} ; 1-\varepsilon<\left|w_{1}\right|<1+\varepsilon,\left|w_{\imath}\right|<1+\varepsilon \quad(\imath=2,3, \cdots, n)\right\}, \\
& D\left(\varepsilon, \varepsilon^{\prime}\right)=\left\{w=\left(w_{1}, w_{2}, \cdots, w_{n}\right) \in \boldsymbol{C}^{n} ;\left|w_{1}\right|<1+\varepsilon-\varepsilon^{\prime},\left|w_{\imath}\right|<1-\varepsilon^{\prime}(\imath=2,3, \cdots, n)\right. \\
& \cup\left\{w=\left(w_{1}, w_{2}, \cdots, w_{n}\right) \in \boldsymbol{C}^{n} ; 1-\varepsilon+\varepsilon^{\prime}<\left|w_{1}\right|<1+\varepsilon-\varepsilon^{\prime},\left|w_{\imath}\right|<1+\varepsilon-\varepsilon^{\prime}(\imath=2,3, \cdots, n)\right\}
\end{aligned}
$$

and

$$
E(\varepsilon)=\left\{w=\left(w_{1}, w_{2}, \cdots, w_{n}\right) \in \boldsymbol{C}^{n} ;\left|w_{\imath}\right|<1+\varepsilon(\imath=2,3, \cdots, n)\right\} .
$$

Then there are a positive number $\varepsilon$ and a biholomorphic mapping $\tau$ of $E(\varepsilon)$ in $C^{n}$ such that $\tau(D(\varepsilon))$ is a subdomain of $\Omega$, that there is a point $a=\left(a_{1}, a_{2}, \cdots, a_{n}\right)$ of $C^{n}$ such that its mage $\tau(a)$ is a boundary point of $\tau(D(\varepsilon))$ and $\Omega$ at the same time and that it satisfies $\left|a_{1}\right| \leqq 1-\varepsilon,\left|a_{2}\right|=1,\left|a_{\imath}\right|<1+\varepsilon(\imath=3,4, \cdots, n)$. Since the $L$-regularity and the $p_{7}$-convexity which is a local property are invariant under the analytic isomorphism $\tau$, we may assume that $\tau$ is the identity mapping of $E(\varepsilon)$. We put

$$
H=\left\{\left(w_{1}, w_{2}, 0, \cdots, 0\right) \in \boldsymbol{C}^{n} ;\left(w_{1}, w_{2}\right) \neq\left(a_{1}, a_{2}\right)\right\} .
$$

There are strictly monotonically decreasing sequences $\left\{\varepsilon_{p}\right\}$ and $\left\{\delta_{p}\right\}$ of positive numbers such that $D^{\prime}\left(\varepsilon_{p}\right)=D\left(\varepsilon, \varepsilon_{p}\right) \subset \Omega_{p}$ and the set

$$
G_{p}=\left\{w=\left(w_{1}, w_{2}, \cdots, w_{n}\right) \in \Omega_{p} ;\left|w_{\imath}\right|<\delta_{p}(i=3,4, \cdots, n)\right\}
$$

satisfies $\left(w_{1}, w_{2}\right) \neq\left(a_{1}, a_{2}\right)$ for any point $\left(w_{1}, w_{2}, \cdots, w_{n}\right)$ of $G_{p}$ and that $\varepsilon_{p} \rightarrow 0$ and $\delta_{p} \rightarrow 0$ as $p \rightarrow \infty$.

Let $\mathfrak{U}=\left\{U_{\imath} ; i \in I\right\}$ be any open covering of $H$ and $\left\{f_{\imath j}\left(w_{1}, w_{2}\right)\right\}$ be any element of $Z^{1}\left(\mathfrak{H}, \mathcal{A}_{L}\right)$. We define an open covering $\mathfrak{B}_{p}=\left\{V_{\imath}^{p}\right\}$ of $G_{p}$ for any $p$ by putting

$$
V_{\imath}^{p}=\left\{w=\left(w_{1}, w_{2}, \cdots, w_{n}\right) \in G_{p} ;\left(w_{1}, w_{2}, 0, \cdots, 0\right) \in U_{\imath}\right\} .
$$

for any $\imath \in I$. Then $\left\{f_{\imath j}\left(w_{1}, w_{2}\right)\right\}$ defines an element of $Z^{1}\left(\mathfrak{B}_{p}, \mathcal{A}_{L}\right)$. Since $\Omega_{p}$ is $L$-regular in $C^{n}$, we have $H^{1}\left(G_{p}, \mathcal{A}_{L}\right)=0$. There is an element $\left\{g_{\imath}^{p}\right\}$ of $C^{0}\left(\mathfrak{V}^{p}, \mathcal{A}_{L}\right)$ 
for any $p$ such that

$$
f_{\imath j}\left(w_{1}, w_{2}\right)=g_{j}^{p}\left(w_{1}, w_{2}, \cdots, w_{n}\right)\left(g_{i}^{p}\left(w_{1}, w_{2}, \cdots, w_{n}\right)\right)^{-1}
$$

in $V_{\imath}^{p} \cap V_{\jmath}^{p}$ for any $i, \jmath \in I$. We put

and

$$
\mathfrak{U}^{p}=\left\{V_{\imath}^{p} \cap D^{\prime}\left(\varepsilon_{p}\right) \cap H ; \imath \in I\right\}
$$

$$
\mathfrak{U} \cap D(\varepsilon) \cap H=\left\{U_{i} \cap D(\varepsilon) \cap H ; \imath \in I\right\}
$$

for any $p$. Then $\mathfrak{H}^{p}$ is an open covering of $D^{\prime}\left(\varepsilon_{p}\right) \cap H$ and $\left\{g_{i}^{p}\left(w_{1}, w_{2}, 0, \cdots, 0\right)\right\}$ is an element of $C^{0}\left(\mathfrak{U}^{p}, \mathcal{A}_{L}\right)$ such that

$$
f_{\imath \jmath}\left(w_{1}, w_{2}\right)=g_{\jmath}^{p}\left(w_{1}, w_{2}, 0, \cdots, 0\right)\left(g_{i}^{p}\left(w_{1}, w_{2}, 0, \cdots, 0\right)\right)^{-1}
$$

in $V_{\imath}^{p} \cap V_{\jmath}^{p} \cap D^{\prime}\left(\varepsilon_{p}\right) \cap H$. Since $\left\{D^{\prime}\left(\varepsilon_{p}\right) \cap H ; p=1,2,3, \cdots\right\}$ is a monotonically increasing sequence of analytically contractible open sets in $C^{2}$ and since $D(\varepsilon) \cap H$ is its limit, by Lemma 1 there is an element $\left\{f_{\imath}\right\}$ of $C^{0}\left(\mathfrak{H} \cap D(\varepsilon) \cap H, \mathcal{A}_{L}\right)$ such that

$$
f_{\imath j}=f_{\jmath} f_{\imath}^{-1}
$$

in $U_{i} \cap U_{j} \cap D(\varepsilon) \cap H$ for any $i, j \in I$.

Now we continue to prove Lemma 4 . If $L$ is abelian, by the previous paper [11] of the author and Kazama, the limit $\Omega$ of $L$-regular domains $\Omega_{p}$ is a Stein manifold. So we may assume that $L$ is a non-abelian connected $m$-dimensional complex Lie group. Let $\mathcal{G} \mathcal{L}(m, C)$ and $\mathcal{L}$ be, respectively, the Lie algebras of $G L(m, C)$ and $L$. Let $\exp : \mathcal{G} \mathcal{L}(m, \boldsymbol{C}) \rightarrow G L(m, \boldsymbol{C})$ and $\exp : \mathcal{L} \rightarrow L$ be the exponential mappings. Let $a d: \mathcal{L} \rightarrow \mathcal{G} \mathcal{L}(m, \boldsymbol{C})$ and $A d: L \rightarrow G L(m, \boldsymbol{C})$ be the adjoint representations. We have

$$
A d \exp (t X)=\exp (\operatorname{tad} X)
$$

for any $t \in C$ and $X \in \mathcal{L}$. Since $L$ is not abelian, there is an element $X$ of $\mathcal{L}$ such that

$$
B=\operatorname{ad} X
$$

is a non-zero $(m, m)$-matrix. We consider an open covering $\mathfrak{U}=\left\{H_{1}, H_{2}\right\}$ of the $H=\left\{w=\left(w_{1}, w_{2}, 0, \cdots, 0\right) \in \boldsymbol{C}^{n} ;\left(w_{1}, w_{2}\right) \in \boldsymbol{C}^{2}-\left\{\left(a_{1}, a_{2}\right)\right\}\right\}$ defined by

$$
H_{i}=\left\{w=\left(w_{1}, w_{2}, 0, \cdots, 0\right) \in \boldsymbol{C}^{n} ; w_{i} \neq a_{\imath}\right\} \quad(i=1,2) .
$$

Two cases may occur. In case that $B$ has a non-zero eigen-value, we put

$$
k\left(w_{1}, w_{2}\right)=\frac{1}{\left(w_{1}-a_{1}\right)\left(w_{2}-a_{2}\right)}
$$

in $H_{1} \cap H_{2}$. And, in case that all eigen-values of $B$ are zero, we put

$$
k\left(w_{1}, w_{2}\right)=\exp \left(\frac{1}{w_{1}-a_{1}}+\frac{1}{w_{2}-a_{2}}\right)
$$


in $H_{1} \cap H_{2}$. Then, in each case, $k$ is a holomorphic function in $H_{1} \cap H_{2}$. Therefore $\exp \left(k\left(w_{1}, w_{2}\right) X\right) \in H^{0}\left(H_{1} \cap H_{2}, \mathcal{A}_{L}\right)$ defines an element of $Z^{\mathfrak{1}}\left(\mathfrak{H}, \mathcal{A}_{L}\right)$. By the above argument, there are $f_{1} \in H^{0}\left(H_{1} \cap D(\varepsilon), \mathcal{A}_{L}\right)$ and $f_{2} \in H^{0}\left(H_{2} \cap D(\varepsilon), \mathcal{A}_{L}\right)$ such that

$$
\exp \left(k\left(w_{1}, w_{2}\right) X\right)=f_{2} f_{1}^{-1}
$$

ih $H_{1} \cap H_{2} \cap D(\varepsilon)$. We put

$$
g_{i}=\operatorname{Ad} f_{\imath}
$$

in $H_{i} \cap D(\varepsilon)(\imath=1,2)$. Then $g_{1} \in H^{0}\left(H_{1} \cap D(\varepsilon), \mathcal{A}_{G L(m, c)}\right)$ and $g_{2} \in H^{0}\left(H_{2} \cap D(\varepsilon)\right.$, $\left.\mathcal{A}_{G L(m, C)}\right)$ satisfy

$$
\exp \left(k\left(w_{1}, w_{2}\right) B\right)=g_{2} g_{1}^{-1}
$$

in $H_{1} \cap H_{2} \cap D(\varepsilon)$. Hence each element of the matrix $g_{2}$, det $g_{2}$ and $1 / \operatorname{det} g_{2}$ are holomorphic functions in $\left\{\left(w_{1}, w_{2}, 0, \cdots, 0\right) \in \boldsymbol{C}^{n} ;\left|w_{1}\right|<1+\varepsilon,\left|w_{2}\right|<1\right\} \cup\left\{\left(w_{1}, w_{2}, 0\right.\right.$, $\left.\cdots, 0) \ni \boldsymbol{C}^{n} ; 1<\left|w_{1}\right|<1+\varepsilon, w_{2} \neq a_{2}\right\} . \quad g_{2}$ is continued to an element of $H^{0}\left(H_{2} \cap E(\varepsilon)\right.$, $\left.\mathcal{A}_{G L(m, c)}\right)$. Hence

$$
g_{1}=\exp \left(-\frac{B}{\left(w_{1}-a_{1}\right)\left(w_{2}-a_{2}\right)}\right) g_{2}
$$

is holomorphic in $\left\{\left(w_{1}, w_{2}, 0, \cdots, 0\right) \in \boldsymbol{C}^{n} ;\left|w_{2}\right|<1+\varepsilon, 1<\left|w_{1}\right|<1+\varepsilon\right\} \cup\left\{\left(w_{1}, w_{2}, 0\right.\right.$, $\left.\cdots, 0) \in \boldsymbol{C}^{n} ;\left|w_{2}\right|<1+\varepsilon, w_{2} \neq a_{2},\left|w_{1}\right|<1+\varepsilon, w_{1} \neq a_{2}\right\}$. Hence $g_{1}$ is continued to an element of $H^{0}\left(H_{1} \cap E(\varepsilon), \mathcal{A}_{G L(m, c)}\right)$. Each $g_{\imath}$ is continued analytically to an element of $H^{0}\left(H_{i} \cap E(\varepsilon), \mathcal{A}_{G L(m, c)}\right)$. Since $C^{2} \times\{(0,0, \cdots, 0)\} \cap E(\varepsilon)$ is an open neighborhood of $\left(a_{1}, a_{2}, 0, \cdots, 0\right)$ in $\boldsymbol{C}^{2} \times\{(0,0, \cdots, 0)\}$ and since $\left(H_{1} \cap E(\varepsilon)\right) \cup\left(H_{2} \cap E(\varepsilon)\right)$ $=C^{2} \times\{(0,0, \cdots, 0)\} \cap E(\varepsilon)-\left\{\left(a_{1}, a_{2}, 0, \cdots, 0\right)\right\}$, this contradicts to Lemma 2 or Lemma 3.

Q.E. D.

\section{§ 3. L-regular domain with smooth boundary.}

An open subset $G$ of a complex manifold $M$ is said to have smooth boundary if for any point $x^{0}$ of the boundary $\partial G$ of $G$ in $M$ there are a neighborhood $V$ of $x^{0}$ in $M$ and a real-valued differentiable function $g$ in $V$ such that

and $\operatorname{grad} g \neq 0$ in $V$.

$$
\partial G \cap V=\{x \in V ; g(x)=0\}
$$

THEOREM. Let $L$ be a complex Lie group with positive dimension. Let $D$ be an L-regular domain with smooth boundary in a Stein manifold $S$. Then $D$ is a Stern manifold.

Proof. Let $s^{0}$ be any boundary point of $D$ in $S$. There are $n$ holomorphic functions $z_{1}(s), z_{2}(s), \cdots, z_{n}(s)$ in $S$ such that they form a local coordinate system in a neighborhood $V$ of $x^{0}$ and that $z_{i}\left(s^{0}\right)=0(i=1,2, \cdots, n)$ where $n$ is the dimension of $S$. For a sufficiently small $\varepsilon$, we put

$$
U=\left\{s \in V ;\left|z_{i}(s)\right|<\varepsilon\right\}
$$


and we may assume that there is a real-valued differentiable function $g$ in variables $z_{1}, z_{2}, \cdots, z_{n-1}, y_{n}$ such that

$$
\partial D \cap U=\left\{s \in V ; x_{n}=g\left(z_{1}, z_{2}, \cdots, z_{n-1}, y_{n}\right)\right\}
$$

where $x_{n}$ and $y_{n}$ are, respectively, the real and imaginary parts of $z_{n}$. It suffices to consider the case that

$$
D \cap U=\left\{s \in V ; x_{n}<g\left(z_{1}, z_{2}, \cdots, z_{n-1}, y_{n}\right)\right\} .
$$

For $0 \leqq t<1$ we put

$$
\begin{aligned}
& E_{t}=\left\{s \in V ; x_{n}<g\left(z_{1}, z_{2}, \cdots, z_{n-1}, y_{n}\right)-t \varepsilon / 2,\right. \\
& \left.\left|z_{\imath}\right|<(1-t) \varepsilon / 2(i=1,2, \cdots, n)\right\} .
\end{aligned}
$$

Then $E_{t}$ is an $L$-regular open set for $0 \leqq t<1$ and $E_{0}$ is exhausted by them. Hence $E_{0}$ is a Stein manifold by Lemma 4 . Therefore $D$ is pseudoconvex in the sense of Cartan, that is, $p_{4}$-convex in the sense of Docquier-Grauert [4]. Therefore $D$ is a Stein manifold by Docquier-Grauert [4].

\section{REFERENCES}

[1] Behnke, H. Und K. Stein, Analytische Funktionen mehrerer Veränderlichen $\mathrm{zu}$ vorgebenen Null- und Pol-stellenflähen. Jber. D. M. V., 47 (1937), 177-192.

[2] Cartan, H., Les problèmes de Poincaré et de Cousin pour les fonctions de plusieurs variables complexes. C. R. Paris, 198 (1934), 1284-1287.

[3] CARTAN, H., Sur les premières problèmes de Cousin. ibid., 207 (1938), 558-560.

[4] Docquier, F. und H. Grauert, Levisches Problem und Rungescher Satz für Teilgebiete Steinscher Mannigfaltıkeit. Math. Ann., 140 (1960), 94-123.

[5] Grauert, H., Analytische Faserungen über holomorph-volständigen Räume. Math. Ann., 135 (1958), 263-273.

[6] Kajiwara, J., On Thullen's example of Cousin-II domain. Sci. Rep. Kanazawa Univ., 9 (1964), 1-8.

[7] Kajiwara, J., Some characterizations of Stein manifold through the notion of locally regular boundary points. Kōdai Math. Sem. Rep., 16 (1964), 191198.

[8] KaJiwara, J., On the limit of a monotonous sequence of Cousin's domains. J. Math. Soc. Jap., 17 (1965), 36-46.

[9] KaJiwara, J., Relations between domains of holomorphy and multiple Cousin's problems. Kōdai Math. Sem. Rep., 17 (1965), 261-272.

[10] Kajiwara, J., Some extension of Cartan-Behnke-Stein's theorem. Pub. Res. Inst. Math. Sc1. Kyoto Un1v., 2 (1966), 133-156.

[11] Kajiwara, J. AND H. Kazama, Two dimensional complex manifold with vanishing cohomology set. Math. Ann., 204 (1973), 1-12.

[12] OKA, K., Sur les fonctions analytıques de plusieurs variables. II. Domaine d'holomorphie. J. Sc1. Hiroshıma Univ., 7 (1937), 115-130. 
[13] OкA, K., - III. Deuxième problème de Cousin. ibid., 9 (1939), 7-19.

[14] Ока, K., IX. Domaines finıs sans point critıque intérieur. Jap. J. Math., 23 (1953), 97-155.

[15] Thullen, P., Sur deuxième problème de Cousin. C.R. Paris, 200 (1935), 720 721.

[16] Thullen, P., Bemerkungen über analytische Flächen in Raume von $\mathrm{n}$ kom. plexen Veränderlichen. Math. Ann., 183 (1969), 1-5.

Department of Mathematics, Faculty of Science, KYUSHU UNIVERSITY. 